\title{
Real-Time Adaptive Foreground/Background Segmentation
}

\author{
Darren E. Butler \\ Information Security Institute, Queensland University of Technology, Brisbane QLD 4001, Australia \\ Email:de.butler@qut.edu.au
}

V. Michael Bove Jr.

Media Laboratory, Massachusetts Institute of Technology, Cambridge, MA 02139, USA

Email:vmb@media.mit.edu

\author{
Sridha Sridharan \\ Information Security Institute, Queensland University of Technology, Brisbane QLD 4001, Australia \\ Email: s.sridharan@qut.edu.au
}

Received 2 January 2004; Revised 8 November 2004

\begin{abstract}
The automatic analysis of digital video scenes often requires the segmentation of moving objects from a static background. Historically, algorithms developed for this purpose have been restricted to small frame sizes, low frame rates, or offline processing. The simplest approach involves subtracting the current frame from the known background. However, as the background is rarely known beforehand, the key is how to learn and model it. This paper proposes a new algorithm that represents each pixel in the frame by a group of clusters. The clusters are sorted in order of the likelihood that they model the background and are adapted to deal with background and lighting variations. Incoming pixels are matched against the corresponding cluster group and are classified according to whether the matching cluster is considered part of the background. The algorithm has been qualitatively and quantitatively evaluated against three other well-known techniques. It demonstrated equal or better segmentation and proved capable of processing $320 \times 240$ PAL video at full frame rate using only $35 \%-40 \%$ of a $1.8 \mathrm{GHz}$ Pentium 4 computer.
\end{abstract}

Keywords and phrases: video segmentation, background segmentation, real-time video processing.

\section{INTRODUCTION}

As humans we possess an innate ability to decompose arbitrary scenes and with only a casual glance we can recognise a multitude of shapes, shades, and textures. In contrast, computers require enormous amounts of processing power and frequently fail if, for instance, the sun hides behind a cloud. As a consequence, practical solutions often rely upon domain-specific knowledge to make the problem tractable. For instance, if we know that we are compressing a head-andshoulders sequence, then we also know that most of the information pertains to the participant. Hence, we can employ differential bit allocation and encode their facial expressions and gestures with a higher quality than the background. Alternatively, we could try to fit a parameterised model to the participant [1] or derive one from them [2] and thereby obtain even greater compression. In either case, the first step is to segment the participant from the background and we can exploit their movements to help us do so.

Motion is a particularly important cue for computer vision. Indeed, for many applications, the simple fact that something is moving makes it of interest and anything else can be ignored. In such cases, it is common for moving objects to be referred to as the foreground and stationary objects as the background. A classic example from the literature is automatic traffic flow analysis [3] in which motion is used to differentiate between vehicles (the foreground) and the roadway (the background). Higher-level processing could then be employed to categorise the vehicles as cars, motorcycles, buses, or trucks. Such a system might be used for determining patterns of traffic flow, or it could be adapted to automatically identify traffic law violations. Other applications where motion is important include gesture tracking $[4,5]$, person tracking $[6,7,8]$, model-based video coding $[9,10,11]$, and content-based video retrieval [12]. In practice, the need to segment moving objects from a static background is so common, it has spawned a nichè area of research where it is known as background subtraction, background segmentation, or background modelling.

As a priori knowledge of a scene's background does not often exist, the key for any background segmentation algorithm is how to learn and model it. The simplest approach 
involves calculating an average background frame whilst no moving objects are present. Subsequently, when objects enter the scene, they will cause the current frame to diverge from the background frame and their presence can be easily detected by thresholding the difference between them [13]. However, any background or illumination change will severely and indefinitely degrade the accuracy of the algorithm. Therefore, practical implementations must continuously update the background frame to incorporate any permanent scene changes. Furthermore, assuming that the background is perfectly stationary is also flawed. For instance, a tree branch waving in the wind moves but is typically not important and so should be incorporated into the background model. We define pseudostationary backgrounds as having constituent objects that are either motionless or that undergo small repetitive motions. A single average background frame is clearly incapable of correctly modelling pseudostationary backgrounds. In practice, the stationarity assumption is often retained and subsequent processing is used to eliminate errors.

Background segmentation is but one component of a potentially very complex computer vision system. Therefore, in addition to being accurate, a successful technique must consume as few processor cycles and as little memory as possible. An algorithm that segments perfectly but is very computationally complex is useless because insufficient processing power will remain to do anything useful with its results.

Seed and Houghton conducted one of the earliest studies into background subtraction in 1988 [14]. They realised that true background changes tend to occur gradually and hence proposed a number of techniques that refined the background frame accordingly. Of the techniques, the two most promising were random updating and slope limited updating. Random updating replaces background pixels by the corresponding pixels in the current frame according to a pseudorandom sequence. As no reference is made to what data the pixels actually contain, errors in the background frame will occur. However, the errors are isolated and can be reduced using conventional morphological operations. In contrast, slope limited updating only adjusts the background frame when it differs substantially from the current frame and even then only by small amounts. Remarkably, given the level of computer technology at the time, they further showed that by using these techniques, it was possible to distinguish between vehicles and the roadway in real time.

More recently, Chien et al. have revisited background subtraction [15]. They surmised that the longer a pixel remained roughly constant, the more likely it is that it belongs to the background. Pixels are classified as stationary when the amount by which they change between consecutive frames falls below a threshold. Once a pixel has remained stationary for a sufficient number of frames, it is copied into the background frame.

Although the above algorithms succeed in learning and refining the background frame, none of them is capable of handling pseudostationary backgrounds. Stauffer and Grimson recognised that these kinds of backgrounds are inherently multimodal and hence they developed a technique which models each pixel by a mixture of Gaussians [16]. Incoming pixels are compared against the corresponding mixture in an effort to find a Gaussian that is within 2.5 standard deviations. If such a Gaussian exists, then its mean and variance are refined according to the pixel. However, if no match is found, then the minimum weighted Gaussian is replaced by a new one with the incoming pixel as its mean and a high initial variance. Gaussians that are matched more frequently are nearby often occuring pixels and hence they are more likely to model the background. This algorithm has since been updated to suppress shadows and to improve its learning rate [17].

A less obvious advantage of Stauffer and Grimson's technique is its ability to rapidly adapt to transient background changes. For example, if an object enters the scene and stops moving, it will eventually be incorporated into the background model. If it then moves again, the system should recognise the original background as corresponding Gaussians should still remain in the mixture. However, maintaining these mixtures for every pixel is an enormous computational burden and results in low frame rates when compared to the previous approaches. Our algorithm is most similar to that of Stauffer and Grimson but with a substantially lower computational complexity. As we will show, it has the capability of processing $320 \times 240$ video in real time on modest hardware.

The remainder of the paper is organised as follows: Section 2 introduces the new background segmentation scheme and Section 3 details its implementation, including a few subtle optimisations; the postprocessing algorithm is described in Section 4; Section 5 compares our approach with a few other published techniques and presents some results; some real-world applications that have benefitted from this work are outlined briefly in Section 6; and finally, Section 7 concludes the paper.

\section{ALGORITHM}

The premise of our approach is that the more often a pixel takes a particular colour, the more likely it is that it belongs to the background. Therefore, at the heart of our algorithm is a very low complexity method for maintaining some limited but important information about the history of each pixel. To do this, we model each pixel by a group of $K$ clusters where each cluster consists of a weight $w_{k}$ and an average pixel value called the centroid $c_{k}$. A group is necessary because a single cluster is incapable of modeling the multiple modes that can be present in pseudostationary backgrounds. The size of the group should therefore be set according to the expected modality of the background. Empirically we have found that 3 to 5 clusters per group yield a good balance between accuracy and computational complexity.

In developing our algorithm, we also assumed that there was no automatic gain or white balance variations and that there is no global scene motion. This was a conscious design decision and is not a flaw as it may first appear. Automatic gain control and automatic white balance are camera features that are designed to make the captured video more 
visually pleasing. However, they can cause dramatic colour variations between consecutive frames and hence are intolerable by almost all computer vision algorithms. Automatic compensation is also not straightforward because different cameras employ different algorithms and only a portion of the frames may be affected. It is possible to compensate for pan, tilt, and other global scene motions but we considered that to be a preprocessing stage which would detract from the key problem we were trying to solve. Furthermore, for many popular applications, like security and videoconferencing, a fixed camera is the norm. The interested reader may like to consult one of the many optical flow algorithms $[18,19]$.

Step 1 (cluster matching). The first step in segmenting incoming frames is to compare each of their pixels against the corresponding cluster group. The goal is to find the matching cluster within the group that has the highest weight and hence the clusters are searched in order of decreasing weight. We define a matching cluster as one which has a Manhattan distance (i.e., sum of absolute differences) between its centroid and the incoming pixel below a user prescribed threshold, T. A threshold greater than zero is required to tolerate acquisition noise. Higher thresholds should be used with inexpensive cameras, such as webcams, and lower thresholds with more precise $3 \mathrm{CCD}$ cameras. Typically we used thresholds in the range of $[10,25]$.

The Manhattan distance is a very useful metric as it is evaluated using only additions and subtractions and can thus be implemented very efficiently. In contrast, assuming RGB video, the squared Euclidean distance metric would require the evaluation of up to three multiplications and two additions for each of the $K$ clusters and for every pixel in the frame. Given the target of $320 \times 240$ video and setting $K=$ 5 result in, on average, 576000 multiplications per frame. Clearly, eliminating these multiplications is very beneficial in terms of reducing the computational complexity of the algorithm.

Step 2 (adaptation). If, for a given pixel, no matching cluster could be found within the group, then the cluster with the minimum weight is replaced by a new cluster having the pixel as its centroid and a low initial weight ( 0.01 in our implementation). The initial weight corresponds with the likelihood that a new cluster belongs to the background. As such, it should be set according to how dynamic the background is expected to be. Rapidly changing backgrounds can have a higher initial weight, whereas it should be decreased if the background is more stationary.

If a matching cluster was found, then the weights of all clusters in the cluster group are updated using

$$
w_{k}^{\prime}= \begin{cases}w_{k}+\frac{1}{L}\left(1-w_{k}\right), & k=M_{k}, \\ w_{k}+\frac{1}{L}\left(0-w_{k}\right), & k \neq M_{k},\end{cases}
$$

where $M_{k}$ is the cluster index of the matching cluster. The parameter $L$ is simply the inverse of the conventional learning rate, $\alpha$, and can be used to control how quickly scene changes are incorporated into the background model. Smaller values for $L$ will result in faster adaptation and larger values result in slower adaptation.

The centroid of the matching cluster must also be adjusted according to the incoming pixel. Previous approaches have adjusted the centroid based on a fraction of the difference between the centroid and the incoming pixel. However, doing so results in fractional centroids and inefficient implementations. We chose instead to accumulate the error between the incoming pixel and the centroid. When the error term exceeds $L-1$, the centroid is incremented, and when it is below $-L$, the centroid is decremented. This is approximately equivalent to adjusting the centroid on every frame using $c_{k}^{\prime}=c_{k}+(1 / L)\left(x_{t}-c_{k}\right)$ but avoids the need for fractional centroids and can be implemented very efficiently as is detailed in Section 3.

Step 3 (normalisation). The weight of a cluster corresponds with how many times it has been matched. Hence, it is from these weights that we can infer information about the history of pixel values. If the weight is high, we know that the pixel has often exhibited a colour similar to the centroid, and according to our premise, the cluster is more likely to model the background. Conversely, if the weight is low, the centroid colour has not appeared very often and the cluster probably models the foreground. This observation can be formalised by ensuring the weights of the cluster group always total to one. Then the weights represent the proportion of the background accounted for by each cluster and hence can be treated somewhat like probabilities. Consequently, after adaptation, the weights are normalised according to

$$
w_{k}^{\prime}=\frac{w_{k}}{S}, \quad \forall k, \text { where } S=\sum_{k} w_{k}
$$

This is the same as dictating that the cluster group weight vector, $\vec{w}$, must always be a unit vector (i.e., $|\vec{w}|=1$ ).

Step 4 (classification). The incoming pixels are classified by summing the weights of all clusters that are weighted higher than the matched cluster. This calculation is simplified by sorting the normalised clusters in order of increasing weight. Sorting the clusters is also necessary for matching then with pixels of the next frame. However, care must be taken to remember the new location of the matched cluster. After sorting, we employ the following trivial calculation:

$$
P=\sum_{k>M_{k}}^{K-1} w_{k}
$$

The result, $P$, is the total proportion of the background accounted for by the higher weighted clusters and is an estimate of the probability of the incoming pixel belonging to the foreground. Larger values of $P$ are evidence that the pixel belongs to the foreground and smaller values are evidence that it belongs to the background. This value can be thresholded 


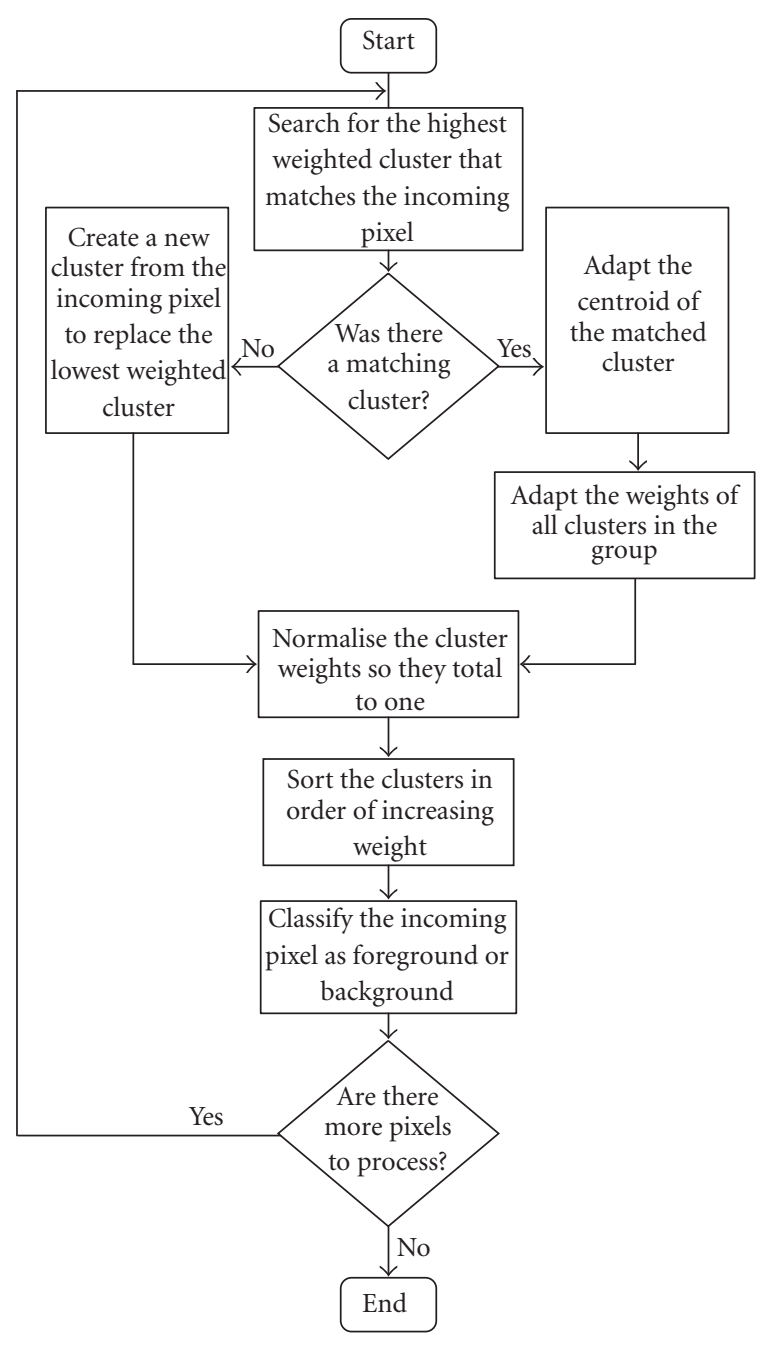

FIgURE 1: The algorithm.

to obtain a binary decision or can be scaled to produce a grayscale alpha map. A pictorial representation of the algorithm is given in Figure 1.

\section{IMPLEMENTATION}

When implementing the algorithm of Section 2, we were conscious of the fact that $Y^{\prime} C_{B} C_{R}$ 4:2:2 video (where $C_{B}$ and $C_{R}$ have been subsampled horizontally by a factor of 2 ) is frequently used when acquiring live video. Fortunately, it is also trivial to upsample to $4: 2: 2$ the $Y^{\prime} C_{B} C_{R}$ 4:2:0 video that is found in popular compression standards like MPEG-2 and H.263. Using $Y^{\prime} C_{B} C_{R}$ 4:2:2 video necessitated making minor modifications to the basic algorithm. Clusters are formed with both a luminance centroid and a chrominance centroid. The luminance centroid consists of two adjacent luminance components $\left(Y_{1}^{\prime}, Y_{2}^{\prime}\right)$ and the chrominance centroid holds the corresponding chrominance components $\left(C_{B}, C_{R}\right)$. Our implementation uses a cluster structure that is similar to that of Algorithm 1. The use of two centroids is beneficial as it allows the specification of different thresholds for luminance

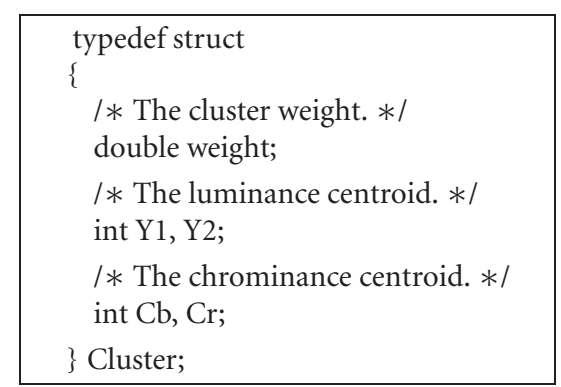

Algorithm 1: The cluster structure.

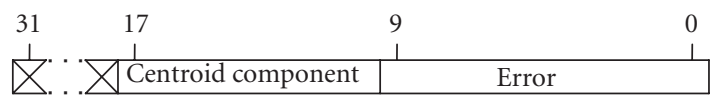

Figure 2: Centroid component bit assignment: $[B=9 \Leftrightarrow L=512]$.

and chrominance. Given only a single threshold, it is likely that luminance would dominate the matching process. With this modification, a match is defined to occur only when the Manhattan distance for both the luminance and chrominance components is below their respective thresholds.

The update equations for the cluster weights are unchanged but we employ a trick to efficiently accumulate the error terms and update the centroids. As aforementioned, the error term lies in the range $[-L, L-1]$. We can equivalently shift this range to $[0,2 \times L-1]$ by the addition of $L$. That is, we simply shift the origin of the error term from 0 to $L$. If we now restrict $L$ to be of the form $L=2^{B}$, then the entire range of the error term can be specified in $B+1$ bits. Furthermore, adaptation of the centroid now occurs when accumulation of the error term results in overflow or underflow of the $B+1$ bits.

Only 8 bits are required to specify any of the components of $Y^{\prime} C_{B} C_{R}$ video. Consequently, if we represent the components of the centroids $\left(Y_{1}^{\prime}, Y_{2}^{\prime}, C_{B}, C_{R}\right)$ by 32 bit integers, then the remaining 24 bits can be used to accumulate the error term. This gives a maximum range for $B$ of $[0,23]$ or, equivalently, $L \in\{1,2,4,8, \ldots, 8388608\}$. Recall that $L$ is synonomous with the inverse of the learning rate, $\alpha$. Therefore, by manipulating the number of bits that are used to store $L$, the user can control the centroid adaptation rate. Accordingly, we shift the components of the centroids upwards by $B+1$ bits and use the lower bits to accumulate the error term, which we initialise to the origin $L$ (see Figure 2). By virtue of this formulation, when overflow or underflow of the error term occurs, the centroid is neatly adjusted automatically. The elegance of this approach is evident in Algorithm 2.

A careful examination of the cluster weight update (1) reveals some further optimisations. As shown below, if the weights of a cluster group sum up to one, then after applying the update equation, they will still sum up to one:

$$
\begin{aligned}
\sum_{k} w_{k}^{\prime} & =\sum_{k} w_{k}+\frac{1}{L} \sum_{k} M_{k}-\frac{1}{L} \sum_{k} w_{k} \\
& =1+\frac{1}{L}-\frac{1}{L}=1 .
\end{aligned}
$$




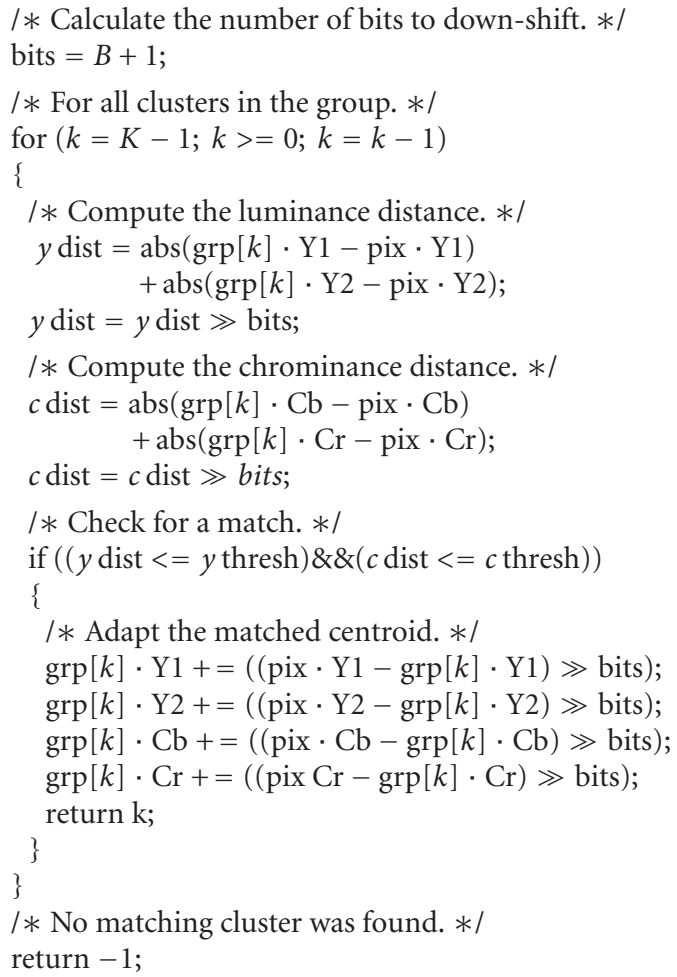

Algorithm 2: FindMatch (ClusterGroup grp, pixel pix).

Therefore, the cluster weights do not need to be normalised unless a matching cluster could not be found and a new cluster had to be created. Furthermore, since the weights of the unmatched clusters are downscaled by exactly the same factor (i.e., $\left.w_{k}^{\prime}=((L-1) / L) \times w_{k}\right)$, only matching clusters and newly created clusters will ever be out of order. As the weights of matched clusters will always increase, the sort operation can be improved by only sorting in the direction of the higher weighted clusters.

The core of our algorithm is the cluster matching operation, pseudocode for which is given in Algorithm 2. The code for adapting the centroid of a matched cluster has been incorporated into the matching code as a subtle optimisation but this is not mandatory. As described in Section 2, cluster matching is based on the Manhattan distance metric because of its simplicity. In fact, it is used so frequently that architecture-dependent optimisations often exist to assist in its calculation. For example, the introduction of the streaming SIMD (single instruction multiple data) extensions (SSE) to Intel's $\mathrm{x} 86$ processors added the $P S A D B W$ instruction. This instruction calculates the sum of the absolute values of the differences between two packed unsigned byte integer vectors of length eight. That is, it computes the Manhattan distance between two unsigned byte integer vectors in one step. More recently, SSE 2 was introduced which extended this capability and allows the simultaneous computation of two different Manhattan distances using 128 bit registers.

In order to understand our approach fully, it is useful to visualise exactly what is being modeled by the clusters.
As aforementioned, each cluster consists of a centroid (i.e., average pixel value), an error term, and a weight, and within their respective cluster groups they are sorted according to their weight. Furthermore, those that are weighted higher have been matched more frequently and consequently are more likely to represent the background. Therefore, if we form images from the centroids at the same position within their cluster group, then intuitively the images should blend from an image of just the background to an image containing the foreground and outdated or noise-ridden centroids. Figure 3 clearly shows that although the blending is not smooth, the images do transition from the background $(k=$ $3)$ to a resemblance of the foreground $(k=0)$ and so inspecting these images is a good method for verifying the correctness of an implementation. Note, however, that this is not the foreground that is returned by the segmenter as foreground pixels are only present in clusters that were just replaced.

\section{POSTPROCESSING}

No practical background segmenter is omniscient. It does not matter how we have formulated it, we cannot guarantee that errors will not occur. In fact, due to the complexity of the problem, the opposite is true: errors are likely. Therefore, it is imperative that steps are taken to detect and eliminate these errors. If we consider background segmentation to be a two-class problem, then logically two kinds of errors can arise. We define false positives to be regions of the background that have been incorrectly labeled as the foreground. Similarly, false negatives are regions of the foreground that have been labeled as the background. The goal of postprocessing is to reduce the number of false postives and false negatives without appreciably degrading the speed of the segmenter.

As shown in Figure 4, false positives usually resemble pepper noise and are often the result of noise in the circuitry of the camera. They are small (1-2 pixel), erroneously classified regions surrounded by correctly classified background pixels. Fortunately, these kinds of errors can be very easily and very quickly eliminated using the morphological open operation. This operation has the additional benefit of smoothing the contour of correctly classified regions without disrupting its shape.

False negatives are typically the result of similarities between the colours of foreground objects and the background. Pixel-based approaches are fundamentally incapable of distinguishing between the foreground and the background if their colours are too similar (the inability of organisms to do this is the basis of camouflage). False negatives typically occur as holes in foreground regions and can be quite large (see Figure 4). They are not as prevalent at the edges of objects because discontinuities are often easier to distinguish. Tightening the luminance and chrominance thresholds (see Section 3) can help to eliminate them but only at the expense of more numerous and more severe false positives. A better approach is to use a connected components algorithm to find connected foreground regions. Small regions are likely to be false positives and can be eliminated. Then, holes in the 


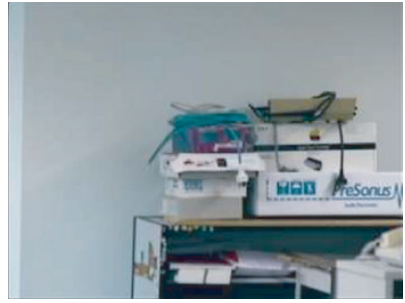

(a)

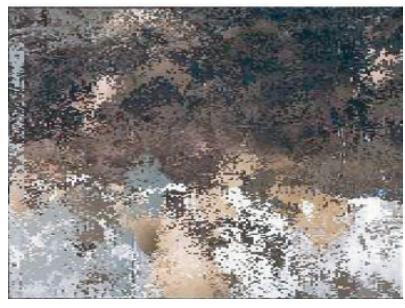

(c)

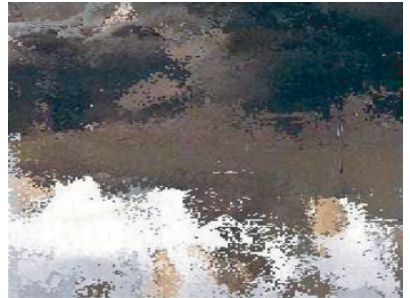

(b)

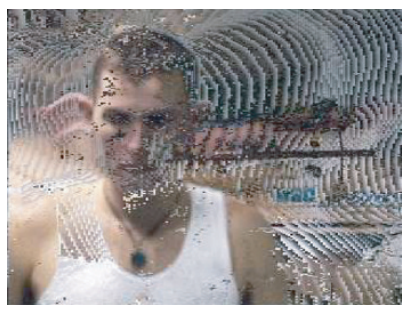

(d)

Figure 3: Centroid images ( $K=4$ and $k$ is the cluster index): (a) $k=3$; (b) $k=2$; (c) $k=1$; and (d) $k=0$.

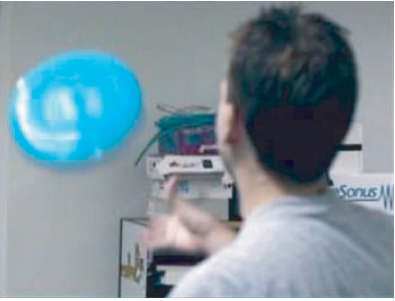

(a)

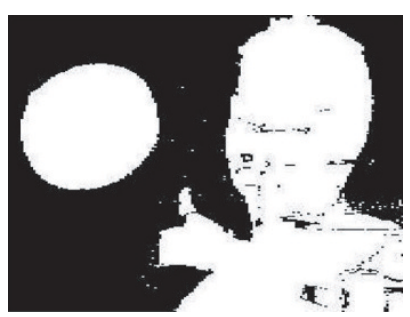

(b)

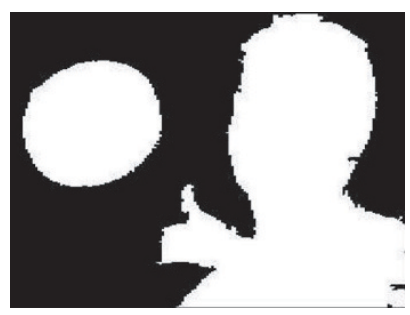

(c)

FIgURE 4: (a) Original; (b) raw segmentation results; and (c) after postprocessing.

remaining regions, which are presumed to be false negatives, can be filled in. Unfortunately, this approach will also fill true holes in the foreground objects, but in practice, this behaviour is often acceptable.

The postprocessor that we utilise is very simple. First, pixels that are separated by a single gap are joined. Next, the contours of all regions that were classified as foreground are extracted using facilities of the Open CV library. ${ }^{1}$ If the area enclosed by the contour is below a user prescribed threshold,

\footnotetext{
${ }^{1}$ The Open Source Computer Vision Library is used courtesy of the Intel Corporation and is available for public download from the World Wide Web at "http://www.intel.com/research/mrl/research/opencv/."
} 
then it can be eliminated. Any remaining contours are presumed to border real foreground objects and are retained. If a binary mask is desired instead, then one can be very easily obtained by filling within the contours. An example of the results obtained with this postprocessor is given in Figure 4 and, as shown, it eliminates most, if not all, of the false positives and the false negatives.

\section{EXPERIMENTAL RESULTS}

We have evaluated our background segmentation algorithm against our implementations of three other techiques. Whilst every effort was made to ensure their correctness, we cannot guarantee that they are completely free of errors. The first method, VAR, calculates an average frame and a variance frame from a buffer of past frames. Each incoming frame is segmented according to the variance normalised difference between it and the average frame. The algorithm, GMM1, is according to the original work of Stauffer and Grimson [16], whereas GMM2 includes the modifications made by KaewTraKulPong and Bowden [17]. Finally, NHD, NHD64, and NHD128 correspond to the C, SSE, and SSE2 versions of our algorithm. The inclusion of assembly language versions may seem unfair but this detail is often very important for embedded systems designers.

It is unreasonable to expect a user to purchase high-end or specialised equipment just to use a particular background segmenter. Consequently, we only used off-the-shelf components when evaluating the four algorithms. Our test-bed consisted of a dual $1000 \mathrm{MHz}$ Pentium 3 computer and a $1.8 \mathrm{Ghz}$ Pentium 4 computer both with $512 \mathrm{MB}$ of RAM. The qualitative analysis was completed using both machines. However, only the Pentium 4 computer was used when collecting the timing and processor utilisation results.

For reasons outlined earlier, the choice of camera was limited to those with manual white balance and manual gain control. We chose to use two cameras: the low-end Bosch $1153 \mathrm{P}$ analog security camera and a more up-market Sony $3 \mathrm{CCD}$ camera. However, for the results presented here, the Sony camera was only used for the stability tests. These cameras both provide a composite output and as such, the captured video was subject to cross-luminance and crosschrominance artifacts, particularly at sharp edges. We found that their effect could be reduced by very slightly defocusing the lens.

\subsection{Qualitative analysis}

Figure 5 visually compares a few manually segmented frames from a five-minute sequence with the results obtained from each of the algorithms. The postprocessor described in Section 4 was used with each of the algorithms and although it performs well in general, it is certainly not optimal in every possible case. Therefore, it is likely that marginally better results could have been obtained by tweaking it but the improvement would have been minimal and would have depended heavily on the acquisition conditions. From the figure, it is clear that our algorithm performs qualitatively as well or better than the other techniques.

\subsection{Quantitative analysis}

In order to quantify the accuracy of each of the algorithms, we segmented 30 seconds of video manually. The video was designed to contain periods of no motion, periods of slow motion, and periods of very fast motion. The fast motion was problematic as it introduced motion blur and was difficult to accurately and consistently segment by hand. Using the video we were able to calculate false acceptance rates (FARs) and false rejection rates (FRRs) for each algorithm, where

$$
\begin{aligned}
& F A R=\frac{\# \text { of false positives }}{\text { \# background pixels }}, \\
& F R R=\frac{\# \text { of false negatives }}{\text { \# foreground pixels }} .
\end{aligned}
$$

Graphs of the FAR and FRR across the entire sequence are given in Figures 6 and 7, respectively. From Figure 6, we can see that, in terms of false acceptances, our algorithm is significantly more accurate than the simple variance-based segmenter. However, it lags behind both of the remaining two segmenters. This may seem to contradict the results of Figure 5 which visually favour our approach but the difference is only around half a percent and is caused by a slight growth in the border of foreground regions.

The false rejection rates for our algorithm are clearly much lower than any of the other techniques. This can also be seen visually by the lack of holes in the segmentation results of Figure 5. Every one of the algorithms exhibits an FRR of $100 \%$ for at least one frame in the sequence. This occurs when the detected foreground region is so small that it is presumed to be noise and is eliminated by the postprocessor. Hence, a large FRR is also an indication that an object has just entered or is about to exit the scene.

\subsection{Computational complexity}

As aforementioned, a key issue for any segmentation algorithm is its computational complexity. It does not matter how accurately it may be able to segment if in doing so it exhausts all of the processor cycles. Table 1 summarises the run-time performance of the four algorithms including the postprocessing stage. For our algorithm, we used a group size of 5 and clearly, given this size, it is approximately two and a half times as complex as the simple variance-based background segmenter. However, its complexity remains well within the capabilities of the hardware and it easily runs in real time ( 25 fps for PAL video). Furthermore, the segmentation results of Figure 5 significantly favour our approach and our background model is adapted on every frame as opposed to the variance-based segmenter which adapts only once every three hundred frames (12 seconds).

\subsection{Stability testing}

The final test for this evaluation was the stability test. This test aimed to determine whether or not a given algorithm would remain convergent when run for a very long time. It was also the only test for which the Bosch security camera was not used as it was unavailable. The test was conducted by 


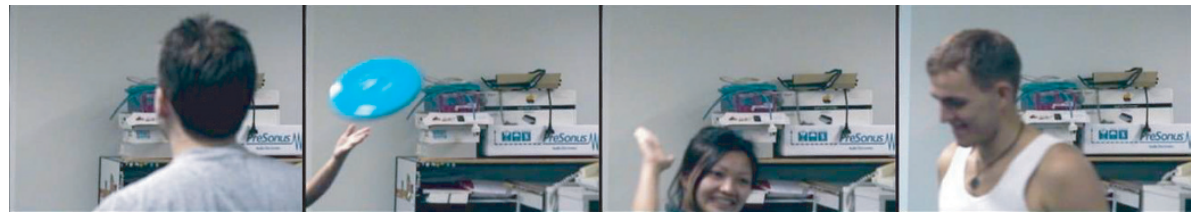

(a)

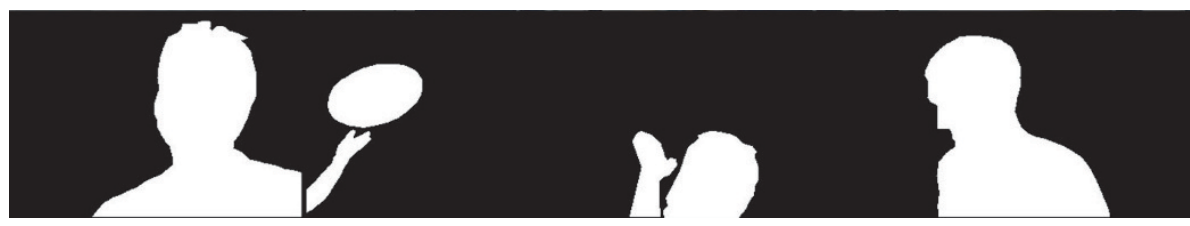

(b)

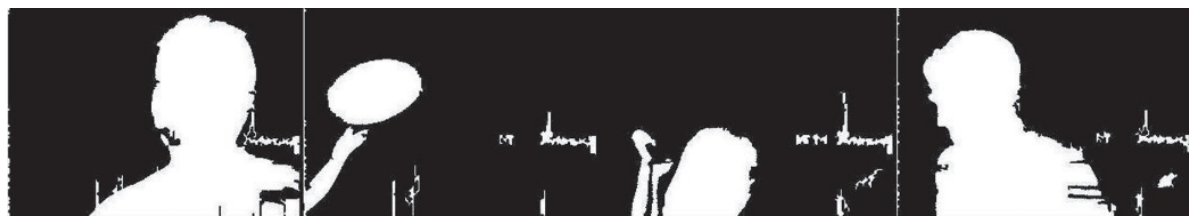

(c)

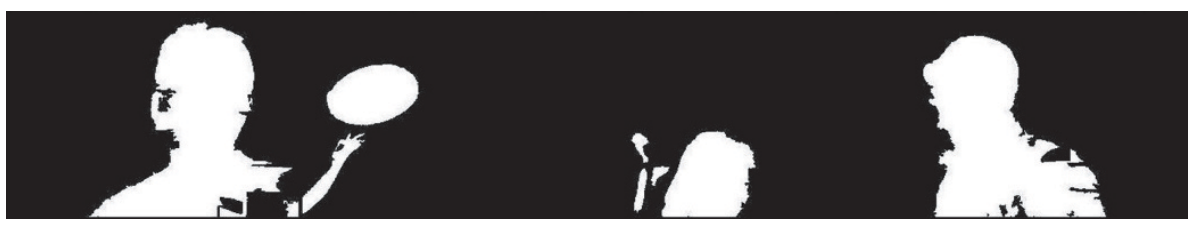

(d)

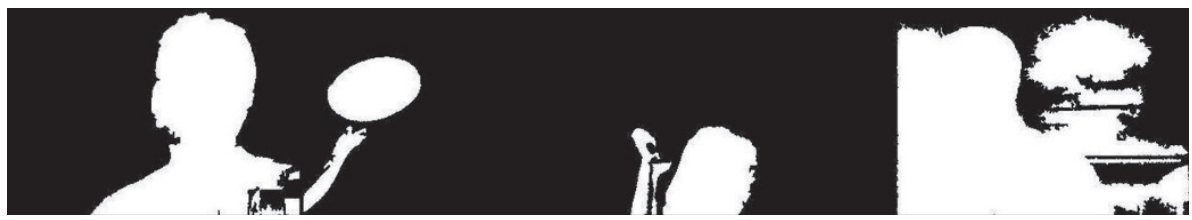

(e)

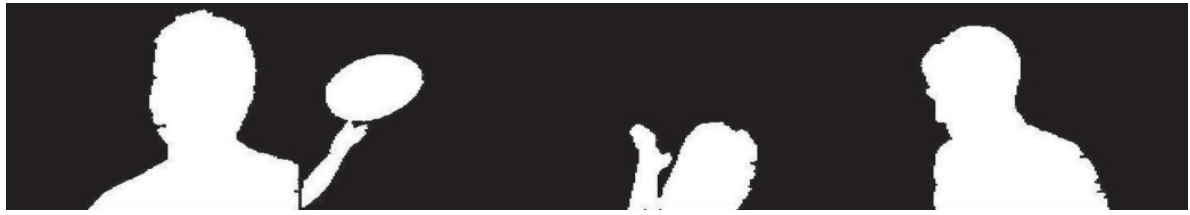

(f)

FIGURE 5: Background segmentation results: (a) original; (b) hand-segmented; (c) VAR; (d) GMM1; (e) GMM2; and (f) NHD/NHD64/NHD128.

connecting the Sony camera to a computer and running our algorithm for a period of approximately twenty-four hours. The background was deliberately arranged to be very cluttered. As is shown in Figure 8, it consisted of nearby objects, far away objects, a glass wall behind which people could walk, and a computer screen that updated itself periodically. The background also exhibited a small degree of pseudostationarity in the form of a suspended sign at the top right of 


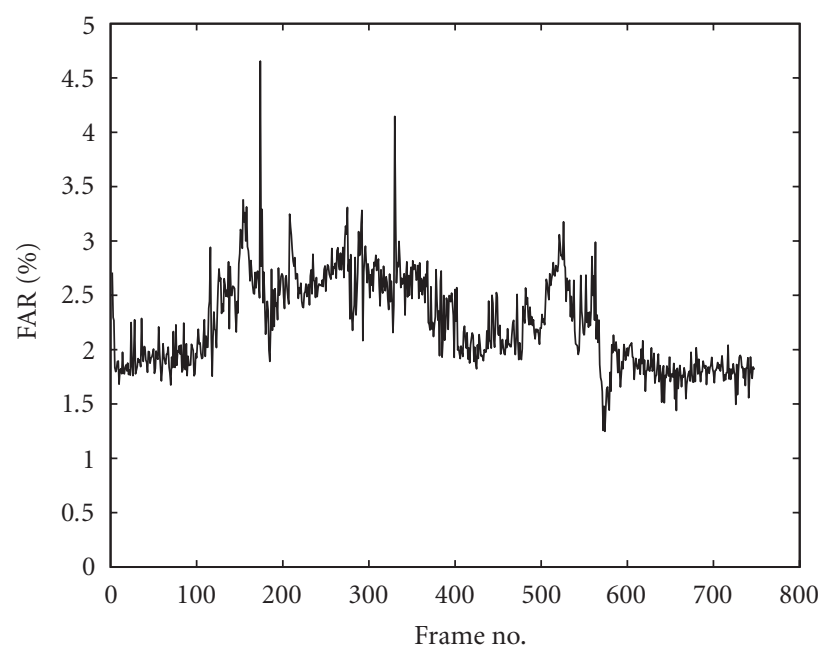

(a)

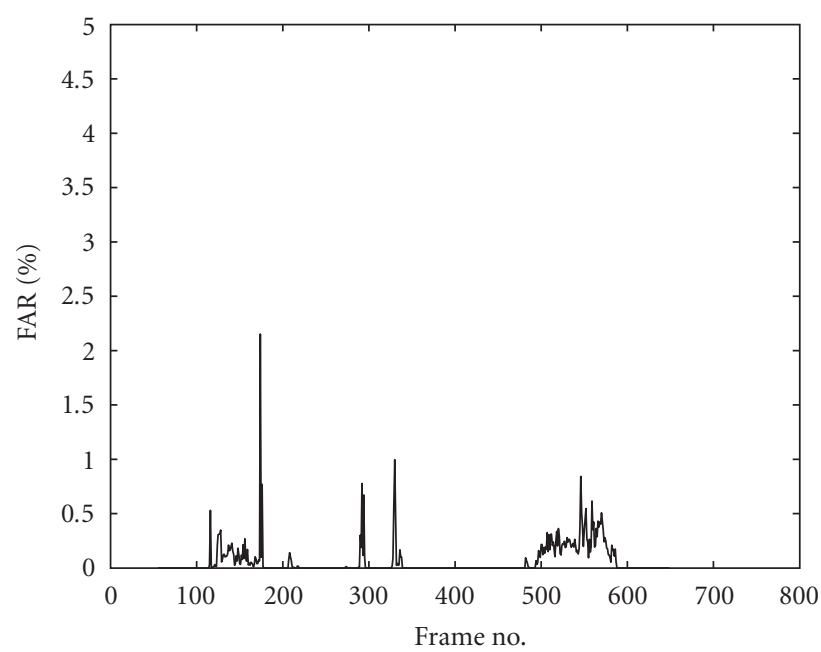

(c)

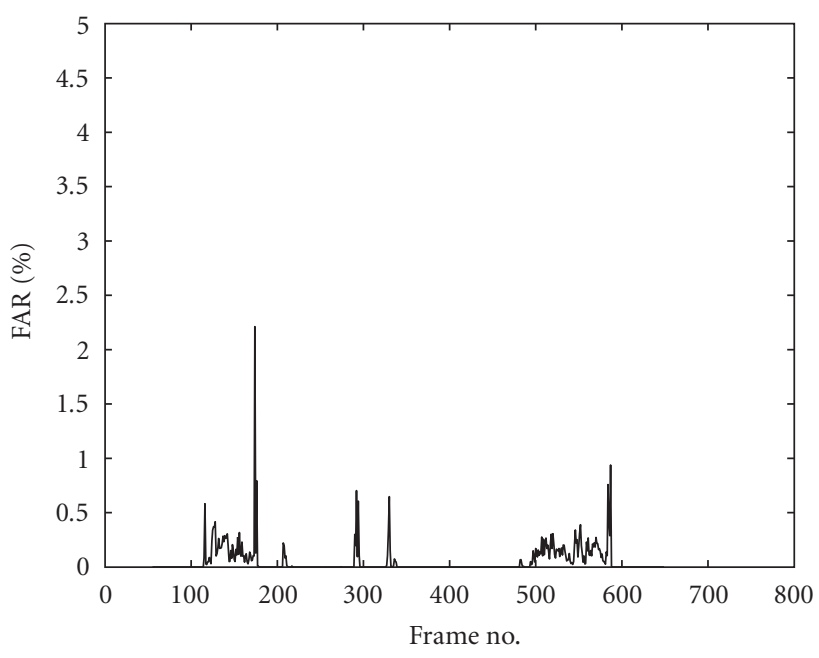

(b)

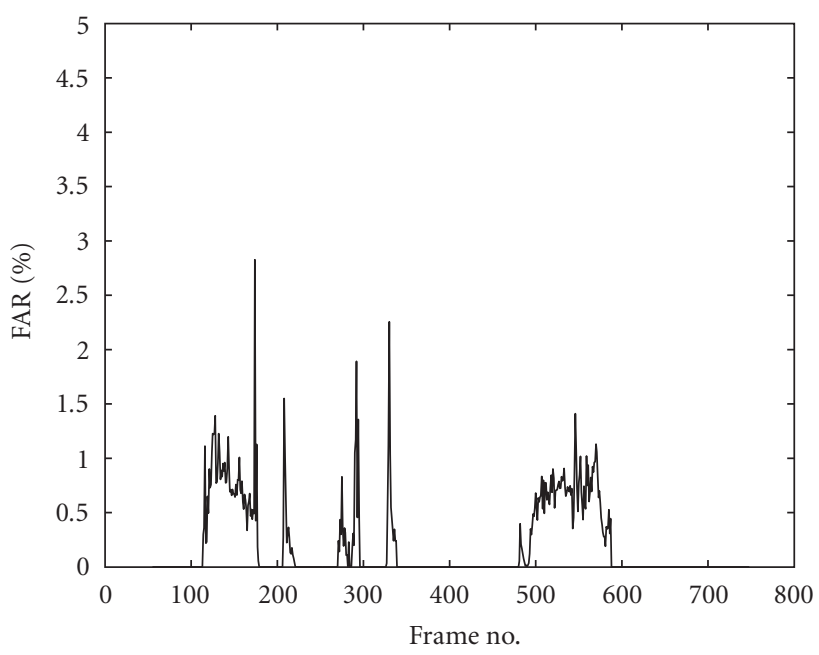

(d)

FIgURE 6: False acceptance rates: (a) VAR; (b) GMM1; (c) GMM2; and (d) NHD/NHD64/NHD128.

the image which fluttered in the wind. In total, over nine hundred thousand (900 000) frames were captured and segmented. However, due to storage constraints, it was not possible to save every frame. Unfortunately, this meant that the likelihood of saving an event of interest was very slim. Nevertheless, Figure 8 contains the segmentation results for frames captured approximately five hours into the test at which time the algorithm is clearly convergent.

\section{APPLICATIONS}

As aforementioned, accurate background segmentation is vital for many interesting applications. Whilst the previous section has proved that our algorithm performs well under laboratory conditions, this does not guarantee it will do so in a real-world application. The major difficulty arises with ensuring that enough hardware resources (CPU cycles and memory) remain after segmentation to do something useful with the results. Therefore, in this section, we briefly describe a few developing applications that exploit our technique and present some preliminary results.

\subsection{Gesture recognition}

When performing an action, a person's body undergoes a signature series of poses. The goal of gesture recognition is to automatically detect these poses and classify the action. Clearly, in order to do this accurately, the person must first be segmented from the background. We have used our algorithm for this purpose in a graffiti detection system that is being developed at the Queensland University of Technology, 


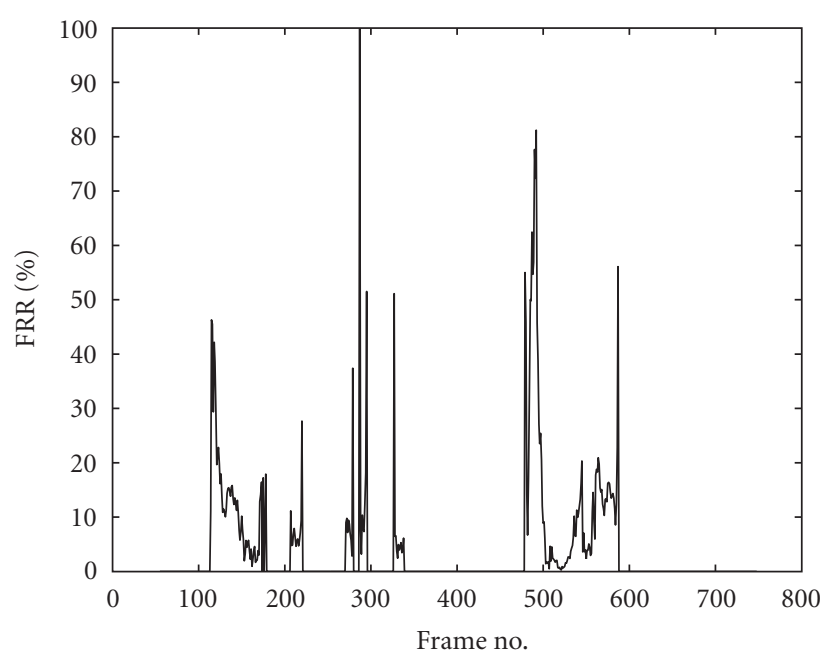

(a)

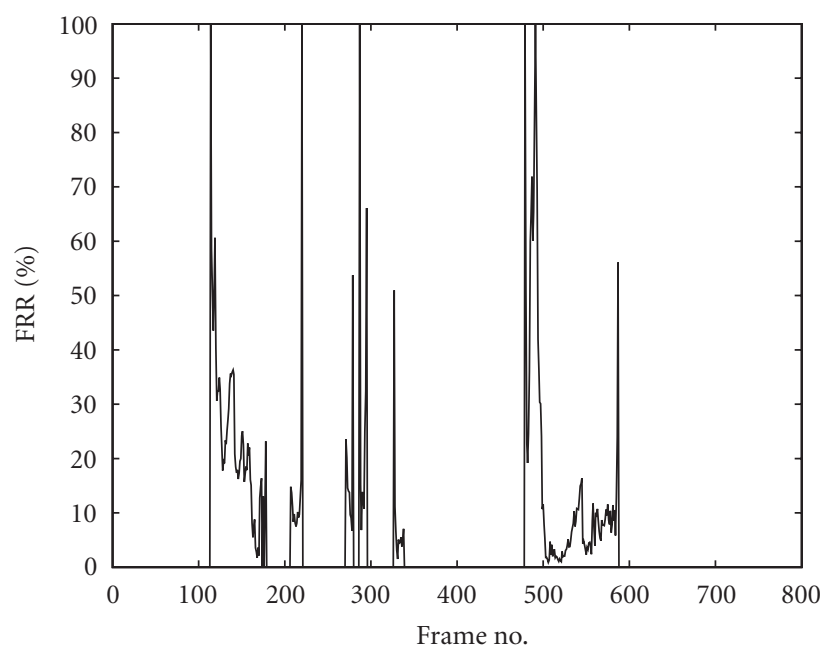

(c)

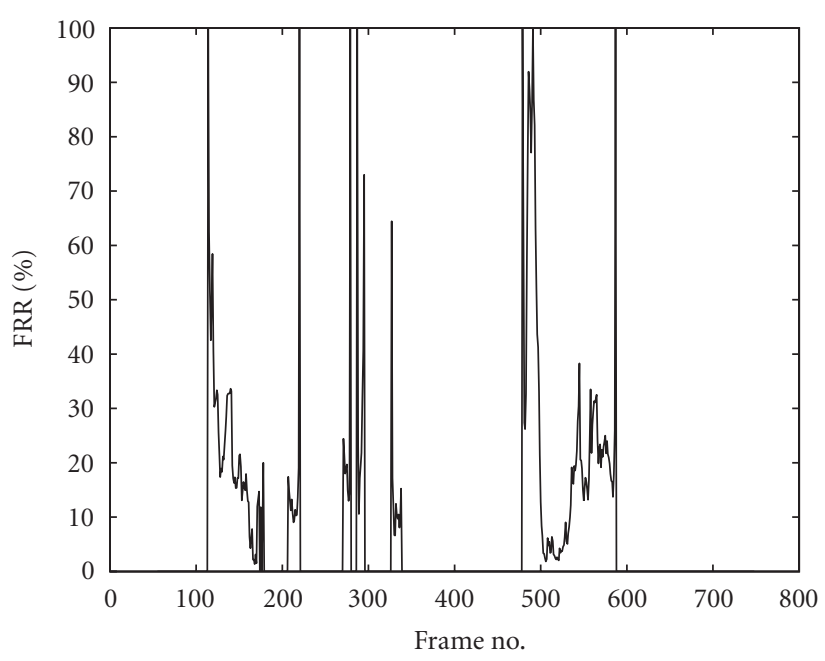

(b)

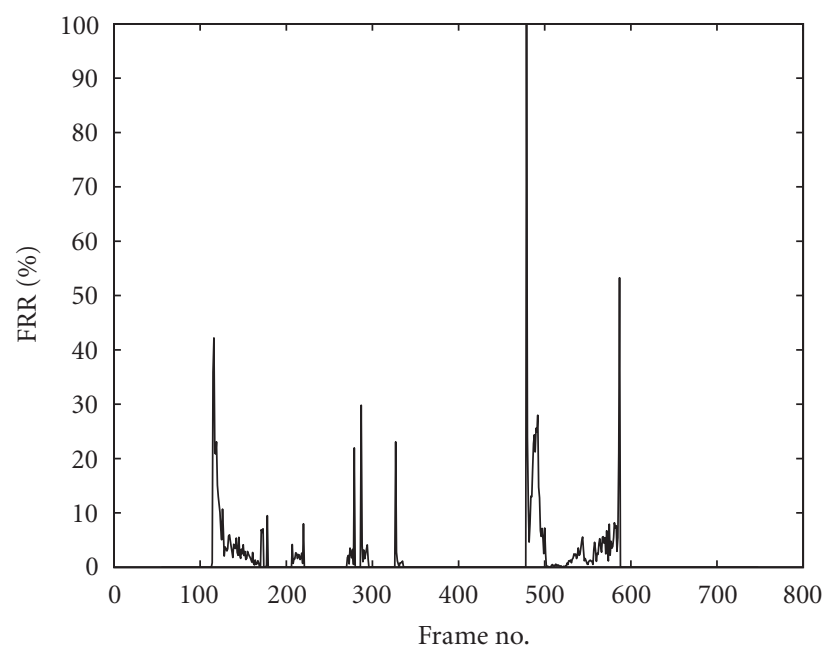

(d)

FiguRE 7: False rejection rates: (a) VAR; (b) GMM1; (c) GMM2; and (d) NHD/NHD64/NHD128.

TABLE 1: Algorithm features and performance.

\begin{tabular}{lccccc}
\hline Algorithm & Video format & User params. & $\begin{array}{c}\text { Frame rate } \\
(\mathrm{fps})\end{array}$ & $\begin{array}{c}\text { Mem. usage } \\
(\mathrm{MB})\end{array}$ & $\begin{array}{c}\text { CPU usage } \\
(\%)\end{array}$ \\
\hline VAR & Y'CbCr & 8 & 25 & 13.5 & 14.4 \\
GMM1 [16] & RGB & 2 & 10 & 25.5 & 99.9 \\
GMM2 [17] & RGB & 2 & $22,13^{\mathrm{a}}$ & 27.0 & 99.9 \\
NHD & Y'CbCr & 4 & 25 & 14.7 & 14.7 \\
NHD64 & Y'CbCr & 4 & 25 & 14.7 & 37.0 \\
NHD128 & Y'CbCr & 4 & 25 & 35.0 \\
\hline
\end{tabular}

${ }^{a}$ The initial transient frame rate of the GMM2 algorithm and its steady-state frame rate, respectively. 


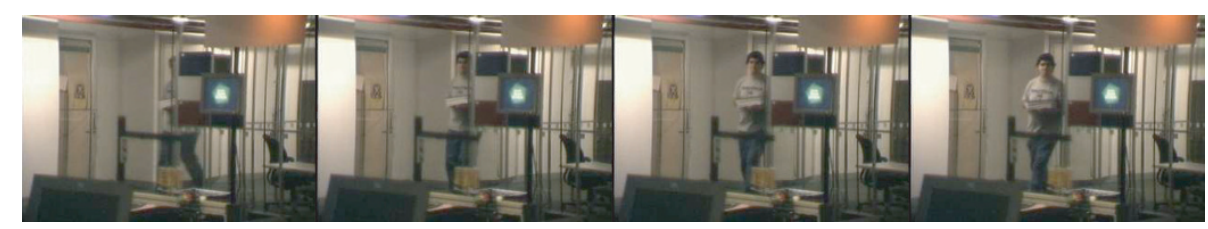

(a)

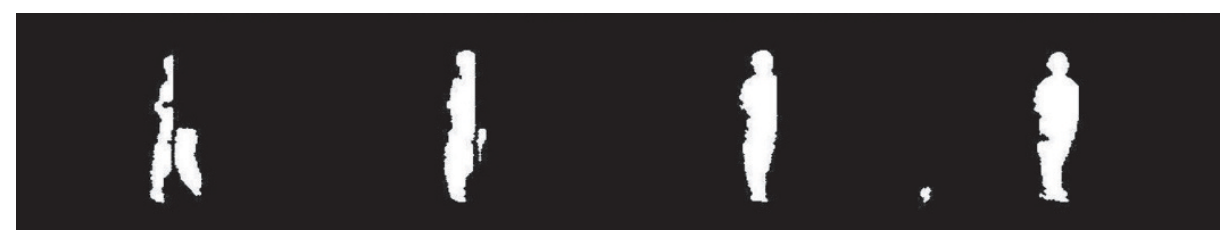

(b)

FIgURE 8: Stability results: (a) original; (b) NHD/NHD64/NHD128.

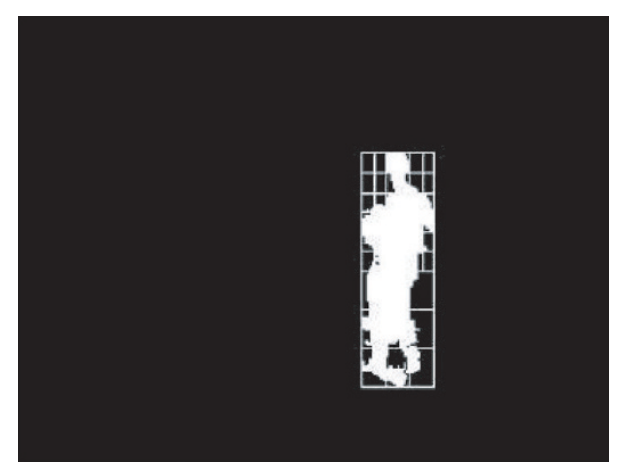

FIGURE 9: The subdivision of the segmentation results in an irregular grid. The ratio of foreground to background pixels in each of the blocks captures the spatial distribution of the body.

Australia [20]. The system is trained to distinguish between passive events and when a person writes on a white board as this action simulates someone writing graffiti.

As depicted in Figure 9, after segmentation, the region of interest is divided into an irregular grid. Since we are interested in human actions, especially those consistent with writing graffiti, the upper portion of the region is more important and hence contains finer subdivisions. Within each block, the proportion of foreground to background pixels is calculated and the resulting ratios are assembled into a feature vector. Vector quantisation is then used to reduce the dimensionality of the feature vector into one of five symbols and the sequence of symbols is classified as a particular event using a discrete hidden Markov model (HMM).

The system was trained using twenty "graffiti" sequences and twenty passive sequences, such as, a person stretching or tying their shoe laces. These sequences were captured in a small room with varying illumination in order to be consistent with real-world conditions. Once trained, the system was used to identify events from continuous video. Preliminary
TABLE 2: Graffiti classification.

\begin{tabular}{lccc}
\hline Sequence & $\begin{array}{c}\text { Number } \\
\text { of events }\end{array}$ & Correctly detected & False alarms \\
\hline${\text { Person } 1^{\text {a }}}$ & 30 & 24 & 12 \\
Unknown1 & 15 & 8 & 7 \\
Unknown2 & 15 & 5 & 4 \\
\hline
\end{tabular}

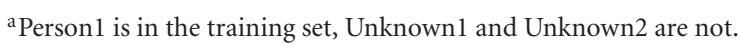

results for video containing people in and out of the training set are summarised in Table 2. Although the algorithm performs quite well when the subject is within the training set, clearly much more work is needed to improve the accuracy when they are not. Shadows are by far the greatest source of error and work is continuing into suppressing them in real time.

\subsection{Interpersonal communications}

A further intended use of the proposed algorithm is as a library function in the Isis multimedia scripting language developed at the MIT Media Laboratory and the Media Lab Europe [21]. This language is used for prototyping networked applications involving video, audio, and graphics on desktop hardware. An example is the reflexion system (previously reflection of presence), Figure 10, a multipoint conferencing and collaboration system which turns each user's screen into a "digital mirror" in which all the users appear. Active participants are composited in the front, while less-active participants are rendered partially transparent behind them. Gesture and object recognition permits users to interact with documents or video streams that make up the background. Other Isis applications under development that require realtime segmentation include systems for recognizing human activity in a space, such as, a meeting room and archiving video of significant events. 


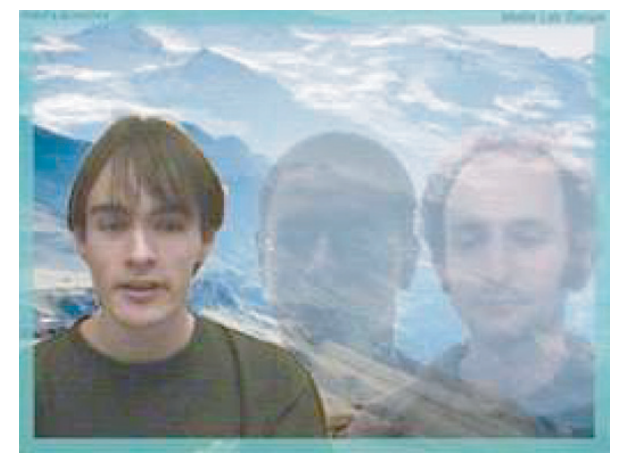

FIgURE 10: Reflexion combines multipoint conference participants in a shared space, a task requiring real-time, visually acceptable segmentation from a desktop PC while leaving enough processing power for audio mixing, object compositing, and other functions (image courtesy Stefan Agamanolis, Media Lab Europe).

\section{CONCLUSIONS AND FUTURE WORK}

The need for fast and accurate algorithms for segmenting moving objects from arbitrary backgrounds is undeniable. The algorithm introduced in this article is a general techique and could be applied to problems as varied as automatic traffic flow analysis and object-based compression. It works by modeling each pixel in the frame by a group of $K$ clusters and then adapting the clusters to account for variations in the background and the ambient conditions. Incoming pixels are compared against the corresponding cluster group according to the Manhattan distance which can be implemented very efficiently. Based on the comparison, a pseudoprobability of the pixel belonging to the foreground is calculated which can be thresholded to obtain a binary decision.

We have evaluated all versions of our algorithm against three other techniques, two of which were drawn from the literature. Our algorithm demonstrated equal or better segmentation than the other techniques whilst being capable of processing $320 \times 240 \mathrm{PAL}$ ( $25 \mathrm{fps}$ ) video in real time, including all postprocessing. Furthermore, to achieve this frame rate, it only utilises between $35 \%-40 \%$ of a $1.8 \mathrm{GHz}$ Pentium 4 computer. In terms of false acceptances, it falls around half a percent behind the Gaussian mixture model-based techniques, but it is consistently better at eliminating false negatives.

The main limitation of our approach is its inability to distinguish between objects and their shadows. When a shadow falls upon a background region, its apparent luminance drops but its chromaticity remains relatively constant [13]. Therefore, it should be possible to enhance the cluster matching process by analysing the direction of the luminance differences as well as their magnitudes. However, at present, shadows either cause the segmentation boundary to blur or in the worst case, they are detected as completely different moving objects. This is a common problem with background segmentation algorithms and future work will look at reducing its impact without significantly increasing the computational complexity of our algorithm.

\section{REFERENCES}

[1] P. Eisert and B. Girod, "Facial expression analysis for modelbased coding of video sequences," in Proc. Picture Coding Symposium (PCS '97), pp. 33-38, Berlin, Germany, September 1997.

[2] D. V. Papadimitriou and T. J. Dennis, “Three-dimensional parameter estimation from stereo image sequences for modelbased image coding," Signal Processing: Image Communication, vol. 7, no. 4-6, pp. 471-487, 1995.

[3] D. Koller, J. Weber, T. Huang, et al., "Towards robust automatic traffic scene analysis in real-time," in Proc. 12th IAPR International Conference on Pattern Recognition (ICPR '94), vol. 1, pp. 126-131, Jerusalem, Israel, October 1994.

[4] F. Quek, X.-F. Ma, and R. Bryll, "A parallel algorithm for dynamic gesture tracking," in Proc. International Workshop on Recognition, Analysis, and Tracking of Faces and Gestures in Real-Time Systems (RATFG-RTS '99), pp. 64-69, Corfu, Greece, September 1999.

[5] A. D. Wilson and A. F. Bobick, "Parametric hidden Markov models for gesture recognition," IEEE Trans. Pattern Anal. Machine Intell., vol. 21, no. 9, pp. 884-900, 1999.

[6] A. Azarbayejani, C. R. Wren, and A. P. Pentland, "Real-time 3-D tracking of the human body," in Proc. IMAGE'COM, Bordeaux, France, May 1996.

[7] C. R. Wren, A. Azarbayejani, T. Darrell, and A. P. Pentland, "Pfinder: real-time tracking of the human body," IEEE Trans. Pattern Anal. Machine Intell., vol. 19, no. 7, pp. 780-785, 1997.

[8] T. C. C. Henry, E. G. Ruwan Janapriya, and L. C. de Silva, "An automatic system for multiple human tracking and actions recognition in office environment," in Proc. IEEE Int. Conf. Acoustics, Speech, Signal Processing (ICASSP '03), vol. 3, pp. 45-48, Hong Kong, China, April 2003.

[9] H. Harashima, K. Aizawa, and T. Saito, "Model-based analysis-synthesis coding of videotelephone images: conception and basic study of intelligent image coding," Transactions of the IEICE, vol. 72, no. 5, pp. 452-459, 1989.

[10] D. E. Pearson, "Developments in model-based video coding," Proc. IEEE, vol. 83, no. 6, pp. 892-906, 1995.

[11] M. Magnor and B. Girod, "Model-based coding of multiviewpoint imagery," in Proc. Visual Communications and Image Processing (VCIP '00), vol. 1, pp. 14-22, Perth, Australia, June 2000.

[12] S.-F. Chang, W. Chen, and H. Sundaram, "VideoQ: a fully automated video retrieval system using motion sketches," in Proc. 4th IEEE Workshop on Applications of Computer Vision (WACV'98), pp. 270-271, Princeton, NJ, USA, October 1998.

[13] T. Horprasert, D. Harwood, and L. S. Davis, "A robust background subtraction and shadow detection," in Proc. 4th Asian Conference on Computer Vision (ACCV '00), Taipei, Taiwan, January 2000.

[14] N. L. Seed and A. D. Houghton, "Background updating for real-time image processing at TV rates," in Image Processing, Analysis, Measurement and Quality, vol. 901 of Proceedings of SPIE, pp. 73-81, January 1988.

[15] S.-Y. Chien, S.-Y. Ma, and L.-G. Chen, "Efficient moving object segmentation algorithm using background registration technique," IEEE Trans. Circuits Syst. Video Technol., vol. 12, no. 7, pp. 577-586, 2002.

[16] C. Stauffer and W. E. L. Grimson, "Adaptive background mixture models for real-time tracking," in Proc. IEEE Computer Society Conference on Computer Vision and Pattern Recognition (CVPR '99), vol. 2, pp. 246-252, Fort Collins, Colo, USA, June 1999.

[17] P. KaewTraKulPong and R. Bowden, "An improved adaptive background mixture model for real-time tracking with 
shadow detection," in Proc. 2nd European Workshop on Advanced Video Based Surveillance Systems (AVBS '01), Kingston, UK, September 2001.

[18] F. Bergholm and S. Carlsson, "A "theory" of optical flow," CVGIP: Image Understanding, vol. 53, no. 2, pp. 171-188, 1991.

[19] H. Tsutsui, J. Miura, and Y. Shirai, "Optical flow-based person tracking by multiple cameras," in Proc. International Conference on Multisensor Fusion and Integration for Intelligent Systems (MFI '01), pp. 91-96, Baden-Baden, Germany, August 2001.

[20] C. Gurrapu and V. Chandran, "Gesture classification using a GMM front end and hidden Markov models," in Proc. 3rd IASTED Conference on Visualization, Imaging, and Image Processing, pp. 609-612, Benalmadena, Spain, September 2003.

[21] S. Agamanolis and V. M. Bove Jr., "Multilevel scripting for responsive multimedia," IEEE Multimedia, vol. 4, no. 4, pp. 4050, 1997.

Darren E. Butler holds a B.IT degree (computer science) and a B.Eng. degree (electronics) both from the Queensland University of Technology, Brisbane, Australia. He has been a Visiting Scholar at the Massachusetts Institute of Technology's Media Laboratory and is the Chief Inventor of a patent relating to video compression and distribution. Darren has received numerous academic awards and is currently consulting for Sarnoff Corporation, NJ, USA, whilst pursuing his Ph.D. degree from the Queensland University of Technology.

V. Michael Bove Jr. holds an S.B.E.E. degree, an S.M. degree in visual studies, and a Ph.D. degree in Media technology, all from the Massachusetts Institute of Technology, where he is currently Head of the ObjectBased Media Group at the Media Laboratory and directs the consumer electronics program CELab. He is the author or coauthor of over 50 journal or conference papers on digital television systems, video process-

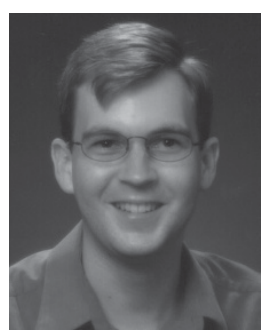
ing hardware/software design, multimedia, scene modeling, visual display technologies, and optics. He holds patents on inventions relating to video recording, hardcopy, interactive television, and medical imaging, and has been a member of several professional and government committees. He is serving as General Chair of the IEEE 2006 Consumer Communications and Networking Conference (CCNC 2006). He is on the Board of Editors of the Journal of the Society of Motion Picture and Television Engineers, and an Associate Editor of Optical Engineering. Bove is a Fellow of the Institute for Innovation, Creativity, and Capital and of the Society of Photo-Optical Instrumentation Engineers. He was a founder of and technical advisor to WatchPoint Media, Inc. (now a part of GoldPocket Interactive).
Sridha Sridharan has a B.Sc. (electrical engineering) degree and obtained an M.Sc. (communication engineering) degree from the University of Manchester Institute of Science and Technology (UMIST), UK, and a Ph.D. degree in the area of signal processing from the University of New South Wales, Australia. He is a Fellow of the Institution of Engineers, Australia, a Senior Member of the Institute of Electrical and

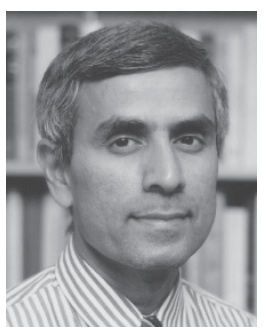
Electronic Engineers-IEEE (USA), and the Chairman of the IEEE Queensland Chapter in Signal Processing and Communication. He is currently with the Queensland University of Technology (QUT) where he is a Professor in the School of Electrical and Electronic Systems Engineering. Professor Sridharan is the Leader of the Research Program in Speech, Audio, Image and Video Technologies (SAIVT) at QUT and is a Deputy Director of Information Security Institute (ISI) at QUT. In 1997, he was the recipient of the Award of Outstanding Academic of QUT in the area of research and scholarship. 